American Journal of Infectious Diseases 4 (1): 32-40, 2008

ISSN 1553-6203

(C) 2008 Science Publications

\title{
Signaling Pathways Involved In Dengue-2 Virus Infection Induced RANTES Overexpression
}

\author{
${ }^{1}$ Ying-Ray Lee, ${ }^{1,2}$ Huan-Yao Lei, ${ }^{2}$ Shun-Hua Chen, ${ }^{3}$ Jen-Reng Wang, ${ }^{2}$ Yee-Shin Lin, ${ }^{3}$ Trai-Ming Yeh, \\ ${ }^{4}$ Ching-Chuan Liu, and ${ }^{2}$ Hsiao-Sheng Liu \\ ${ }^{1}$ Institute of Basic Medical Sciences \\ ${ }^{2}$ Departments of Microbiology and Immunology \\ ${ }^{3}$ Medical Laboratory Science and Biotechnologyand Pediatrics \\ ${ }^{4}$ College of Medicine, National Cheng Kung University
}

\begin{abstract}
Dengue viruses participate in liver inflammation by inducting the expression of various chemokines including Regulated on Activation Normal T-cell Expressed and Secreted (RANTES). However, the underlying signaling remains unknown. Here, we reveal that Ras, Raf-1 and three mitogen-activated protein kinases (MAPKs) p38, extracellular signal-regulated kinase (Erk), and c-jun- $\mathrm{NH}_{2}$-terminal kinase ( $\mathrm{JNK}$ ) can be activated or phosphorylated in dengue-2 virus infected hepatocyte and epithelial cells by western blotting and confirmed by dominant negative mutants of ras, raf-1, p38, Erk, and JNK. The Tet-off inducible plasmids harboring dengue-2 virus prM, core, E or NS1 gene were utilized to reveal their role in RANTES activation. However, no effect was detected among the genes tested indicating that they are either dispensable or not sufficient for RANTES activation. Taken-together, Ras, Raf-1, JNK, Erk and p38 related signaling pathways are essential for the activation of RANTES by dengue-2 virus. The knowledge gathered will shed light on developing a novel therapeutic approach to block inflammatory infiltrates through decreasing RANTES expression.
\end{abstract}

Keywords: RANTES, MAPK signaling pathway, dengue-2 virus

\section{INTRODUCTION}

The mosquito-borne dengue virus (which has four serotypes) is a member of the family Flaviviridae. It occurs predominantly in the tropical and subtropical regions of the world and may cause 100 million cases of dengue fever (DF), 500,000 cases of Dengue Hemorrhagic fever (DHF), and 25,000 deaths. A total of 2.5 billion people are at the risk of dengue virus infection each year ${ }^{[1]}$. The major symptoms are either asymptomatic or self-limited known as DF. The more severe forms of dengue virus infection, DHF and DSS, are characterized by plasma leakage and may be life threatening ${ }^{[2]}$. DHF is a severe febrile disease characterized by abnormalities in homeostasis and increased vascular permeability. Further progression of DHF may result in DSS, which is a form of hypovolemic shock associated clinically with hemonconcentration, which may lead to death. The clinical features include plasma leakage, bleeding tendency, and liver involvement $^{[3,4]}$. Liver involvement is common in dengue virus infected patients with mild elevation of serum transaminases. Dengue virus related dysfunction of hematological, vascular, and hepatic system causes the manifestations of DHF/DSS.

Dengue virus can infect patient liver and cause hepatitis. Elevated serum transaminase levels were found in dengue patients, and the level of AST elevation correlated with that of hemorrhage ${ }^{[5,6]}$. In dengue virus related hepatitis, the level of AST is higher than that of ALT, while the hepatitis induced by other types of viruses have more ALT than AST indicating virus type specific pathogenesis. Dengue virus also can induce apoptosis in human hepatoblastoma cell line $\left.{ }^{[7,} 8\right]$. Previously, Lin et al. ${ }^{[9]}$, demonstrated that RANTES was induced via oxidative stress dependent and independent pathways, which further activates NF-IL6 in dengue-2 virus infected hepatoma cell lines. Moreover, RANTES was preferentially induced in liver cells by dengue virus but not enterovirus and coxsackievirus. Patients with dengue virus infection have increased RANTES serum levels compared to those with other viral infections ${ }^{[9]}$.

Corresponding Author: Hsiao-Sheng Li, Department of Microbiology and Immunolog, College of Medicine, National Cheng Kung University 1 University Road, P.Box 70101,Tainan, Taiwan 
RANTES is a member of the C-C chemokine, which was originally cloned from $\mathrm{CD} 8^{+} \mathrm{T}$ lymphocytes ${ }^{[10]}$. The natural ligands of RANTES, CCR5 chemokine receptor, is the fusion/entry cofactor for M-tropic HIV-1, and RANTES can antagonize the infection of $\mathrm{CD}^{+}$cells to M-tropic strains of HIV-1 ${ }^{[11-13]}$. The importance of RANTES in virus-induced pathogenesis was demonstrated by its role in the pathogenesis of HIV infection. RANTES can recruit lymphocytes and NK cells to the sites of inflammation ${ }^{[14-17]}$. Whether liver damage caused by dengue virus is a direct effect of virus replication or an indirect effect of RANTES-mediated inflammation deserves further investigation. The balance between virus elimination and tissue damage might affect the severity of the disease. Because liver is the major site for the synthesis of most coagulation factors, reduced levels of coagulation factors are either the results of increased consumption or impaired synthesis. The latter is the likely consequence of liver injury.

Many extracellular stimuli elicit specific biological responses through activation of mitogen-activated protein kinase (MAPK) cascades ${ }^{[18]}$. Mammalian MAP kinase superfamily has been molecularly characterized: extracellular signal-regulated kinase (Erk), p38 MAP kinase, and c-Jun- $\mathrm{NH}_{2}$-terminal kinase (JNK) etc. MAP kinase p38 and JNK are activated by environmental stresses including hyperosmotic shock, abnormal temperature, UV irradiation and inflammatory cytokines. They play important roles in apoptosis and cytokine expression $\left.{ }^{[19-24}\right]$. Erk is activated by mitogenic stimuli and is essential for cell proliferation and differentiation $^{[25,26]}$. Erk and JNK also involves in the signaling cascades of various inflammatory mediators including cytokines and chemical mediators ${ }^{[27-32]}$.

RANTES could be activated differently by various stimuli, indicating a wide range of control at the transcriptional level ${ }^{[33-36]}$. In a previous study, HIV infection can activate RANTES through phosphorylation of $\mathrm{Erk}^{[37]}$. And Influenza virus infection can up-regulate RANTES expression through p38 and $\mathrm{JNK}^{[38]}$. Dengue virus can induce DNA binding activity of NF-IL-6 and up-regulates RANTES expression ${ }^{[9]}$. However, whether p38 and JNK are involved in dengue virus related RANTES activation remaining unclear.

All together, the signaling pathways of RANTES activation in dengue- 2 virus infected hepatocyte and endothelial cells were reported in this study.

\section{MATERIALS AND METHODS}

Cell line and virus: Chang liver cells (a non-malignant human liver epithelial cell line) ${ }^{[39]}$, and Hep3B (human hepatoma cell lines) cells were cultured in Dulbecco's modified Eagle's medium, (DMEM; Gibco, USA) supplemented with $10 \%$ fetal bovine serum (FBS; Trace BioSciences, Australia), penicillin (200 units $/ \mathrm{ml}$ ), and streptomycin $(100 \mu \mathrm{g} / \mathrm{ml})$ at $37^{\circ} \mathrm{C}$ in a $5 \% \mathrm{CO}_{2}$ incubator. The human microvascular endothelial cell-1 (HMEC-1) cells were cultured in endothelial basal medium MCDB 131 (GIBCO, USA), supplemented with 10\% FBS (TRACE, Australia), 1\% L-glutamine, $1 \%$ penicillin, hydrocortisone $\left(1 \mu \mathrm{g} \mathrm{mL}^{-1}\right)$ (SIGMA, USA), and $10 \mathrm{ng}$ $\mathrm{mL}^{-1}$ epidermal cell growth factor (EGF) (GIBCO, USA). For virus infection experiments, cells were adsorbed with dengue- 2 virus at the titer of 100 m.o.i. at $37^{\circ} \mathrm{C}$ for $2 \mathrm{hr}$. The cells were then washed three times with phosphate buffered saline (PBS) and incubated at $37^{\circ} \mathrm{C}$ in DMEM (GIBCO-BRL, USA), supplemented with 2\% FBS (TRACE, Australia), penicillin (200 unit/ml) and streptomycin $\left(100 \mu \mathrm{g} \mathrm{mL}^{-1}\right)$.

Dengue-2 virus (PL046 isolated from Taiwan) was maintained in $\mathrm{C} 6 / 36$ cells. The titer was evaluated in BHK-21 cells by plaque assay. Dengue-2 virus was heated at $56^{\circ} \mathrm{C}$ for $30 \mathrm{~min}$ as the inactivated virus ${ }^{[40]}$.

Western blot analysis of p38 MAP kinase, Erk, and JNKL: Analysis of threonine and tyrosine phosphorylation of p38 MAP kinase was performed using an anti-phosphorylated threonine and tyrosine of p38 MAP kinase antibody (anti-phospho-specific p38 MAP kinase Ab, New England Biolabs, USA), which was specific for active p38 MAP kinase and did not cross-react with Erk and JNK. Analysis of threonine and tyrosine phosphorylation of Erk was performed using an anti-phosphorylated threonine and tyrosine of $\mathrm{p} 42 / \mathrm{p} 44$ MAP kinase antibody (anti-phospho-specific p42/p44 MAP kinase Ab, New England Biolabs, USA), which was specific for active p42/p44 MAP kinase and did not cross-react with p38 MAP kinase and JNK. Analysis of threonine and tyrosine phosphorylation of JNK was performed using an anti-phosphorylated threonine and tyrosine of JNK antibody (anti-phospho-specific JNK $\mathrm{Ab}$, New England Biolabs, USA), which was specific for active JNK and did not cross-react with p38 MAP kinase and Erk. Analysis of p38 MAP kinase, Erk, and JNK was performed according to manufacture's instruction. Briefly, after separating proteins from cell lysate by a $15 \%$ SDS-PAGE, the cell lysate containing $10 \mu \mathrm{g}$ of protein was electrophoretically transferred to a 
membrane, and the membrane was incubated with specific antibody to the phosphorylated threonine and tyrosine of p38 MAP kinase (affinity-purified rabbit poly-clonal $\mathrm{IgG}$ ), specific antibody to the phosphorylated threonine and tyrosine of Erk (affinity-purified rabbit poly-clonal $\mathrm{IgG}$ ), or specific antibody to the phosphorylated threonine and tyrosine of JNK (affinity-purified rabbit poly-clonal $\mathrm{IgG}$ ) for analysis of JNK. Then it was incubated with the HRP-conjugated anti-rabbit IgG antibody and HRP-conjugated anti-biotin antibody to detect biotinylated protein markers. Blots were incubated with enhanced chemiluminescence (ECL) solution for $1 \mathrm{~min}$ and exposed on a Kodak (Rochester, NY) XAR film.

Transient transfection and luciferase assay: Cells were cultured in 12-well dish and transfected with pGL-B $\left(1 \mu \mathrm{g}_{\text {well }}{ }^{-1}\right)$, pSG5-lacZ $\left(0.25 \mu \mathrm{g}\right.$ well $\left.{ }^{-1}\right)$, and dominant negative mutant plasmid (DNRas, DNRaf-1, DNErk, DNJNK, DNp38) or vector control $\mathrm{pBSSK}^{+}$ $\left(3 \mu \mathrm{g} \mathrm{well}{ }^{-1}\right)$. The luciferase activities were determined $48 \mathrm{hr}$ after dengue-2 virus infection by a Dual-light luciferase and $\beta$-galactosidase reporter gene assay system (Tropix, Bedford, MA). Briefly, equivalent amounts of protein lysates $(10 \mu \mathrm{l})$ were mixed with buffer A. Light signal emitted from the luciferase enzyme in the extract was measured immediately by a luminometer (Minilumate LB 9506, Germany) after the addition of buffer B containing luciferin and Galacton-Plus. After $30 \mathrm{~min}$ incubation at room temperature, light signal from the accumulated product of $\beta$-galactosidase and Galacton-Plus reaction was elicited by adding of a light emission accelerator and measured by the same luminometer.

Construction of inducible plasmids: The dengue- 2 virus prM, core, NS1 and $\mathrm{E}$ genes in the plasmids pCR-DVMP, pCR-DVCP, pCDNA-NS1 and pCR-DVEP were subcloned into the inducible plasmid vector pTREM2. The expression of pTREM2-DVCP and pTREM2-DVNS1 was confirmed by immunostaining $48 \mathrm{hr}$ after transfection.

\section{RESULTS}

Dengue-2 virus infection induces phosphorylation of MAP kinases, Erk, p38 and JNK: To determine whether dengue-2 virus infection could induce the phosphorylation of three major MAP kinases, Erk, p38

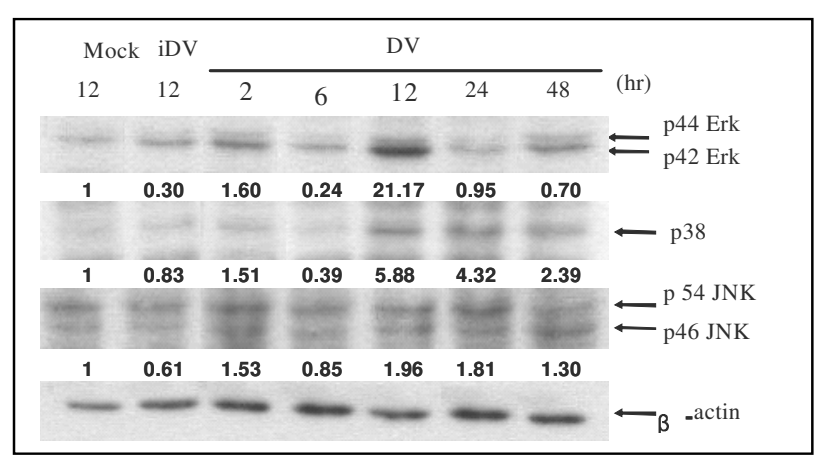

Fig. 1: Phosphorylation of MAPKs in dengue-2 virus infected Chang liver cells by Western blotting. Chang liver cells were infected by dengue- 2 virus for the desired times. The lysates from Chang liver cells were separated by the $15 \%$ SDS-PAGE. The gel was transferred to the membrane and blotted with a specific antibody to phosphorylated JNK. The membrane was stripped and reprobed using a specific antibody to phosphorylated Erk. The membrane was stripped again and reprobed using a specific antibody to phosphorylated p38. Finally, the membrane was stripped and reporbed using a specific antibody of $\beta$-actin as the internal control. To quantify the band intensity, the intensity of MAPK was seated as 1 , all the numbers shown are the comparison with mock

and JNK, Chang liver cells were infected with dengue-2 virus. The levels of phosphorylated Erk (especially p42 Erk) in dengue-2 virus infected cells increased at $2 \mathrm{hr}$ and reached the plateau at $12 \mathrm{hr}$ postinfection (p.i.), and then declined at $24 \mathrm{hr}, 48 \mathrm{hr}$ p.i., respectively (Fig. 1). The level of phosphorylated p38 in dengue-2 virus infected cells was significantly increased at $12 \mathrm{hr}$ p.i., and then slightly declined from 24 to $48 \mathrm{hr}$ p.i. (Fig. 1). Differently, the levels of phosphorylated JNK in dengue-2 virus infected cells were slightly increased at 2 $\mathrm{hr}$.i. and remained at the same level until $48 \mathrm{hr}$ p.i. (Fig. 1). In contrast, heat inactivated dengue viruses suppressed the expression levels of the three MAPKs. Our data demonstrate that active dengue- 2 virus can induce phosphorylation of the three major MAPKs to various levels as well as at different time intervals compared to mock infected cells.

Dengue-2 virus activation of RANTES is through Erk, p38 and JNK signaling pathways: RANTES activation induced by dengue- 2 virus infection has been reported ${ }^{[9]}$. The above data demonstrated that dengue- 2 


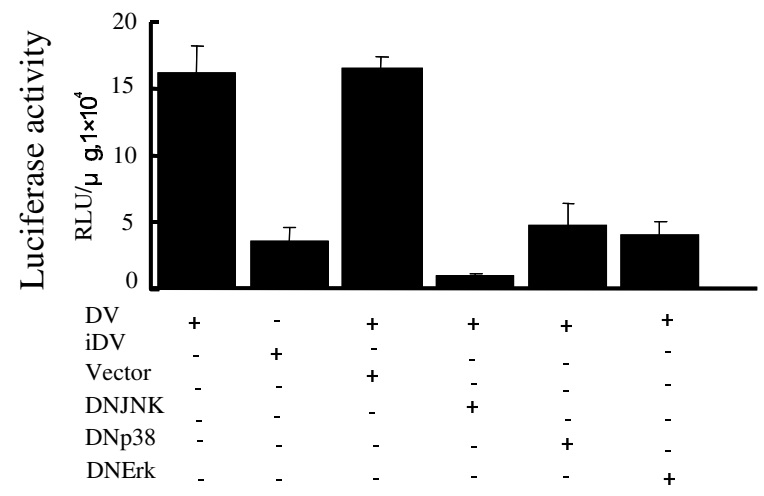

Fig. 2: The effects of dominant negative mutant DNA on RANTES expression in dengue-2 virus infected Chang liver cells by luciferase reporter assay. Chang liver cells were transfected with pGL-B $\left(1 \mu \mathrm{g} \mathrm{well}{ }^{-1}\right)$, pSG5-lacZ $(0.25 \mu \mathrm{g} /$ well $)$, and dominant negative mutants (DNJNK, DNp38, and DNErk) or vector control $\mathrm{pBSSK}^{+}(3 \mu \mathrm{g}$ well ${ }^{-1}$ ) in 12-well culture dish and followed by infection with dengue-2 virus or heat inactived dengue-2 virus (M.O.I.=100). The luciferase activity was measured at $48 \mathrm{hr}$ p.i

virus infection could induce the phosphorylation of Erk, p38 and JNK. It is noteworthy to clarify which of the MAPKs is possibly involved in dengue-2 virus infection-induced RANTES expression. Dominant negative mutants (DN): DNErk, DNJNK, DNp38 for Erk, JNK, and p38, were co-transfected with RANTES reporter plasmid (pGL-B) ${ }^{[9]}$ to block the specific MAPK signaling pathway. RANTES activity was measured by RANTES luciferase reporter analysis at $48 \mathrm{hr}$ p.i.. (Fig. 2) shows that dengue-2 virus infection induced RANTES activity in Chang liver cells was suppressed to the basal level in the presence of DNp38, DNErk or DNJNK. However, cotransfection of RANTES luciferase reporter gene DNA with the empty vector $\left(\mathrm{pBSSK}^{+}\right)$, RANTES activity was not affected. This observation was also seen in Hep3B and HMEC-1 cells suggesting that dengue-2 virus infection-induced RANTES expression in Chang liver, Hep 3B and HMEC-1 cell lines is mediated through Erk-, p38- and JNK-dependent signaling pathways. Moreover, these three MAPKs are not dispensable for dengue virus induced RANTES activation.

Ras and Raf-1 are involved in dengue-2 virus infection-induced RANTES activation: To unveil the up-stream regulator of the MAPK signaling pathways,
A

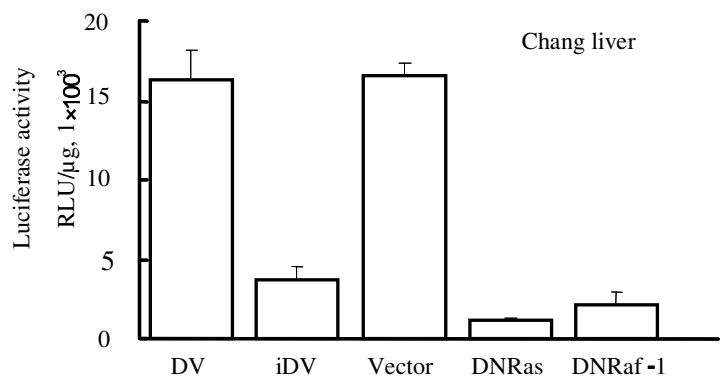

B

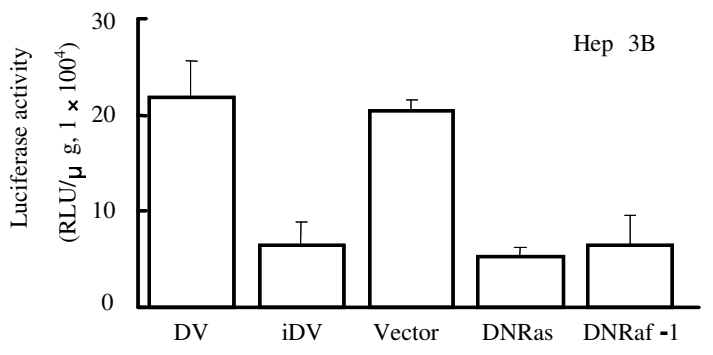

C

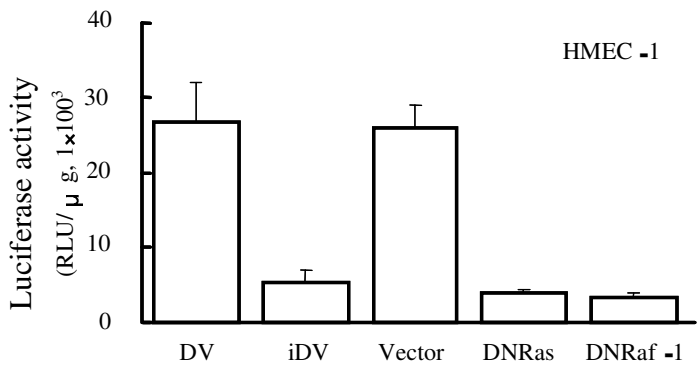

Fig. 3: The effect of dominant negative Ras and Raf-1 on RANTES expression in dengue-2 virus infected cells by luciferase reporter assay. Cells were transfected with pGL-B $\left(1 \mu \mathrm{g}\right.$ well $\left.{ }^{-1}\right)$, pSG5-lacZ $\left(0.25 \mu \mathrm{g}\right.$ well $\left.{ }^{-1}\right)$, and dominant negative mutants (DNRas and DNRaf-1) or vector control $\mathrm{pBSSK}^{+}\left(3 \mu \mathrm{g}\right.$ well $\left.^{-1}\right)$ in 12-well culture dish, followed by infection with dengue-2 virus or heat inactived dengue- 2 virus

(A): Chang liver cells

(B): Hep 3B cells

(C): HMEC-1 cells

Ras/Raf-1 were considered because they are the up-stream transducers of many MAPK signaling pathways. Dominant negative mutant of Ras or Raf-1 (DNRas or DNRaf-1, respectivity) was co-transfected with RANTES reporter plasmid (pGL-B) (Lin et al., 
2000) into Chang liver cells (A), Hep 3B (B) and HMEC-1 (C) cell lines. RANTES activity was measured by RANTES luciferase reporter analysis at $48 \mathrm{hr}$ after dengue-2 virus infection. (Fig. 3) shows that dengue-2 virus induced RANTES activity was declined to the basal level while Ras, or Raf-1 was blocked by DNRas or DNRaf-1. Our data clearly indicate that dengue-2 virus infection-induced RANTES expression is mediated through Ras- and Raf-1-dependent signaling pathways.

A

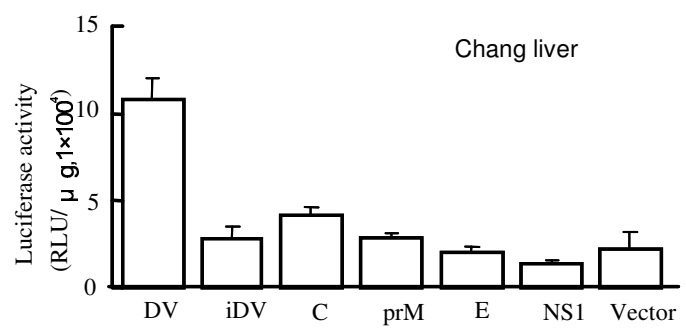

B

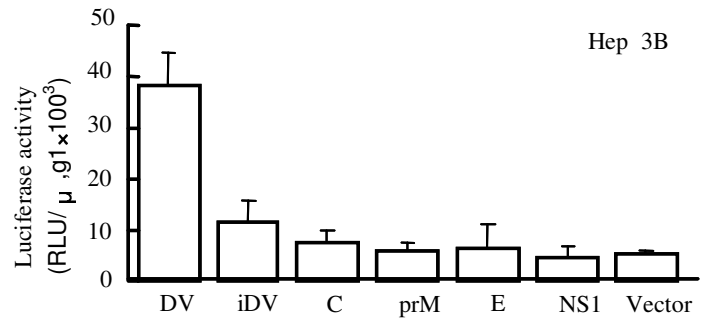

C

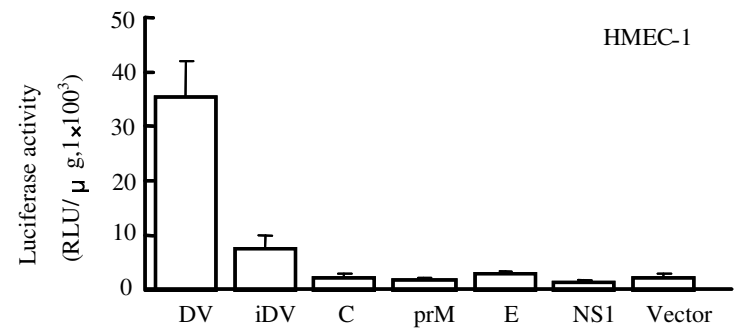

Fig. 4: The effect of single dengue-2 virus gene on RANTES activation in various cell lines. Cells were transfected with pGL-B (1 $\mu \mathrm{g} /$ well $)$, pSG5-lacZ (0.25 $\mu \mathrm{g}$ /well), and dengue-2 virus single gene plasmid (pTREM2-DVCP, pTREM2-DVMP, pTREM2-DVEP, or pTREM2-DVNS1) or vector control pTREM2 (3 $\mu \mathrm{g} /$ well) in 12-well culture dish. The cells were infected with dengue- 2 virus or heat inactived dengue-2 virus (M.O.I.=100). Luciferase activity was measured $48 \mathrm{hr}$ p.i.. (A): Chang liver cells
(B): Hep 3B cells

(C): HMEC-1 cells. C: core protein

Dengue-2 virus prM, core, $\mathrm{E}$ and NS1 proteins are not involved in RANTES activation: To clarify the role of dengue- 2 virus prM, core, E and NS1 protein in RANTES activation, the inducible plasmids harboring dengue-2 virus prM, core, E or NS1 gene were constructed. The expression of the transgenes was detected by immunhistochemistry analysis (data not shown). To determine whether these viral gene products participate in RANTES activation, plasmid: pTREM2-DVCP, pTREM2-DVMP, pTREM2-DVEP, or pTREM2-DVNS1 together with RANTES reporter plasmid (pGL-B) were cotransfected into Chang liver (A), Hep 3B (B) and HMEC-1 (C) cell lines and RANTES activity was measured at $48 \mathrm{hr}$ post-transfection. The RANTES activity was not induced while the cells were transfected with each of the single genes prM, core, E and NS1 as compared to that of dengue-2 virus infected cells (Fig. 4). Our results show that dengue-2 virus single gene prM, core, E or NS1 has no effect on RANTES activation indicating multifactors may be necessary for RANTES activation.

The activation of RANTES expression by dengue-2 virus induced oxidative stress is not regulated by MAPK signaling pathways: According to our findings, we propose a model that dengue-2 virus may up-regulate RANTES expression through Ras/Raf-1/Erk, p38 and JNK signaling pathways (Fig. 6). We previously reported that oxidative stress is essential for

dengue virus induced RANTES activation ${ }^{[9]}$. To reveal the relationship between oxidative stress and MAPK signaling pathways, Chang liver cells pretreated with the pharmaceutical inhibitors PD98059, SB203580 or JNK inhibitor II to block Erk, p38 and JNK-dependent pathways. The $\mathrm{H}_{2} \mathrm{O}_{2}$ expression after dengue-2 virus infection is not affected in pretreated or non-pretreated Chang liver cells by flow cytometry analysis (Fig. 5). Our data indicate that dengue-2 virus induced oxidative stress does not utilized Ras/Raf-1/Erk, p38 and JNK signal pathways to generate $\mathrm{H}_{2} \mathrm{O}_{2}$ and to active RANTES.

\section{DISCUSSION}

In this study, dengue-2 virus infection induced the phosphorylation of three major MAPKs Erk, p38 and JNK at various time points post infection as well as to various levels. Many extracellular stimuli induced maximal MAP kinase phosphorylation and activity within $60 \mathrm{~min}$. These three MAPKs are all activated $2 \mathrm{hr}$ p.i.. The maximal phosphorylation of p38 and Erk was observed at $12 \mathrm{hr}$ after dengue- 2 virus infection. The inconsistency of MAPK activation suggest that 

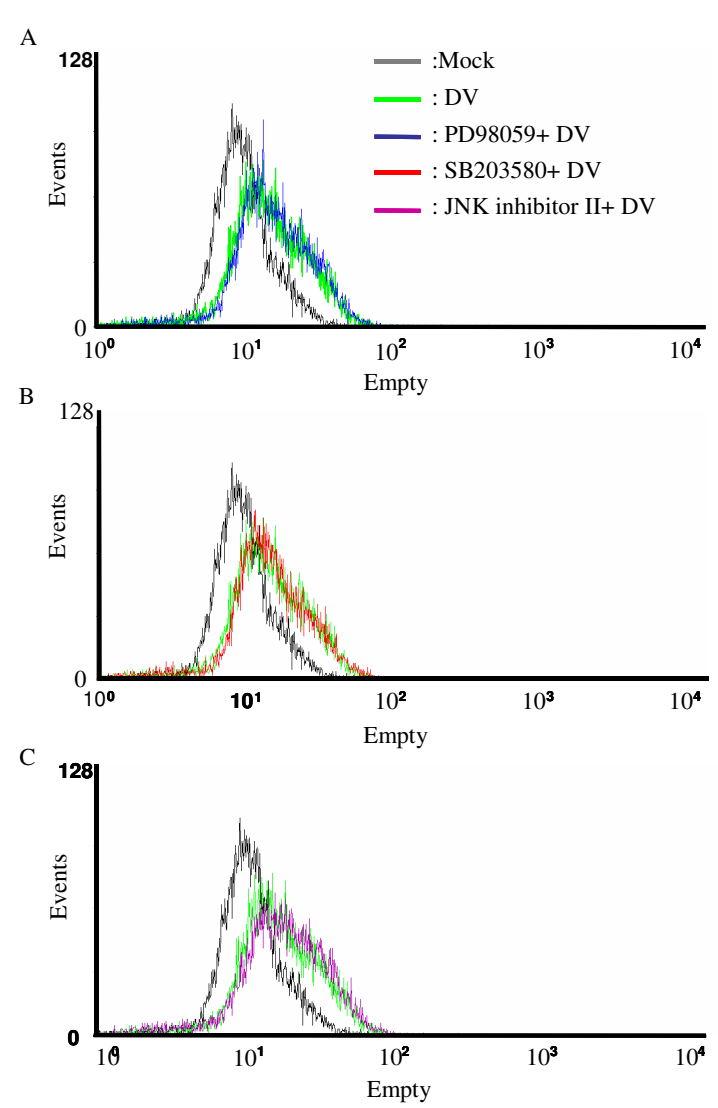

Fig. 5: Measurement of $\mathrm{H}_{2} \mathrm{O}_{2}$ production of Change liver cells after dengue- 2 virus infection. Chang liver cells pre-treated with PD98059 $(50 \mu \mathrm{M})$, SB203580 $(10 \mu \mathrm{M})$, or JNK inhibitor II $(10 \mu$ $\mathrm{M})$ for $2 \mathrm{hr}$ and inoculated with dengue-2 virus (M.O.I.=100). Forty eight hours p.i. 2',7'-dichlorofluorescin diacetate (DCFH-DA, $20 \mu \mathrm{M}$ ) was used to measure $\mathrm{H}_{2} \mathrm{O}_{2}$ production by flow cytometer.

(A) Chang liver cells pretreated with PD98059

(B) Chang liver cells preated with SB203580 (C) Chang liver cells preated with JNK inhibitor II

each signaling pathway may regulate different transcriptional factors, which then act in combination to up-regulate RANTES gene. The RANTES luciferase reporter gene analysis demonstrates that only live dengue- 2 virus could induce RANTES overexpression indicating that the entry and replication of the virus may be involved in RANTES activation (comparing of DV2 and iDV2 infection).

Dominant-negative mutants of Erk, p38 and JNK (DNErk, DNp 38 and DNJNK) further confirm that

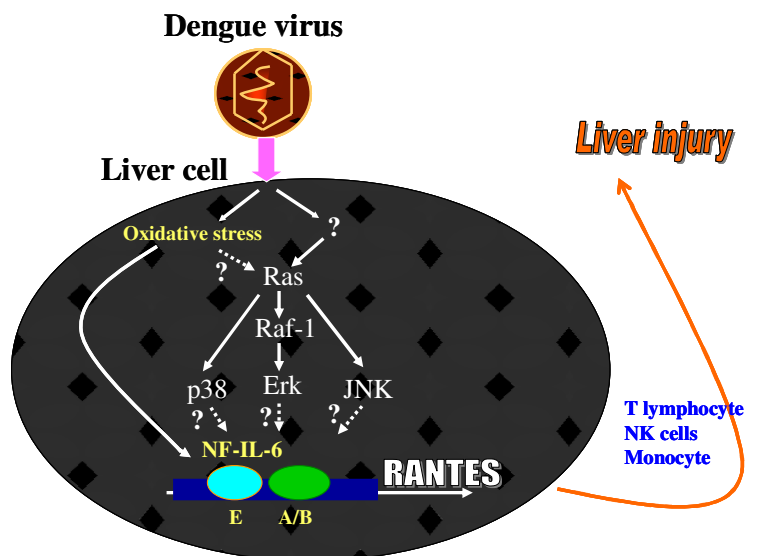

Fig. 6: Diagram of signal transduction of RANTES activation after dengue-2 virus infection. Some connections (the dotted lines) in the diagram are still unclear

dengue-2 virus infection induced RANTES activation is through Erk, p38 and JNK pathways. However, NF-IL6 is the major transcription factor to activate RANTES and the phosphorylation of a threonine residue located at $\mathrm{N}$-terminal of the DNA-binding domain of NF-IL6 by a ras-dependent MAP kinase has been reported ${ }^{[41]}$. Moreover, dengue-2 virus induced RANTES expression is mediated by activation of NF-IL6 through an oxidative stress-dependent and an oxidative stress-independent pathway ${ }^{[9]}$. It is well known that one of the up-stream transducers of Erk signaling pathway is Ras/Raf-1. Consistently, we demonstrated that dengue-2 virus infection utilizes Ras/Raf-1 and Erk, p38 and JNK pathways to induce RANTES activation (Fig. 6).

The kinetics of MAPK phosphorylation is closely correlated with the kinetics of dengue virus replication. We are interested in unraveling whether the single viral gene in dengue-2 virus genome, may be responsible for MAP kinase phosphorylation.

However, none of the core, prM, E and NS1 gene expression could induce RANTES activity indicating the regulation of MAPK phosphorylation is not at signal gene level. Some possibilities can not be excluded in this study. 1. Dengue virus has a total of 10 gene products. Whether other gene products can induce RANTES up-regulation remaining unclear? 2. Other factors may be required together with core, prM, E or NS1 expression to activate RANTES expression. This possibility can be clarified by using dengue- 2 virus infectious cDNA clone, together with site-directed mutagensis to abort the function of specific genes.

Infection of various viruses could also induce RANTES expression in a wide variety of cells ${ }^{[40,42,43]}$. RANTES representing by its chemotactic activity for 
eosinophils plays a pivotal role in the induction of airway inflammation of asthmatics through the recruitment of eosinophils to the inflammation site of airway after influenza virus infection ${ }^{[38]}$. Whether RANTES secretion induced by dengue virus infection of liver cells can recruit immune cells deserves further clarification. Moreover, whether liver damage in dengue patients was caused by a direct effect of virus replication or an indirect effect of RANTES-mediated inflammation needs further investigation. In summary, this study unravels the signaling pathway for RANTES activation in dengue-2 virus infected liver cells and endothelial cells.

\section{ACKNOWLEDGEMENTS}

This work was supported by the grant NHRI-CN-CL9601S from the National Health Reasearch Institutes, ROC.

\section{REFERENCES}

1. Monath, T.P., 1994. Dengue: the risk to developed and developing countries. Proc. Natl. Acad. Sci. USA, 91: 2395-2400.

2. Halstead, S.B., 1989. Antibody, macrophages, dengue virus infection, shock, and hemorrhage: a pathogenetic cascade. Rev. Infect. Dis., 11: S830-839.

3. Bhamarapravati, N., 1989. Hemostatic defects in dengue hemorrhagic fever. J. Infect. Dis., Suppl. 4, S826-S829.

4. Burke, D.S., A. Nisalak, D.E. Johnson and R. M. Scott, 1988. A prospective study of dengue infection in Bangkok. Am. J. Trop. Med. Hyg., 38: 172-180.

5. Kalayanarooj, S., D.W. Vaughn,

S. Nimmannitya, S. Green, S. Suntayakorn, N. Kunentrasai, W. Viramitrachai, S. Ratanachueke, S. Kiatpolpoj, B. L. Innis, A. L. Rothman, A. Nisalak and F. A. Ennis, 1997. Early clinical and laboratory indicators of acute dengue illness. J. Infect. Dis., 176: 313-321.

6. Mohan, B., A.K. Patwari and V.K. Anand, 2000. Hepatic dysfunction in childhood dengue infection. J. Trop. Pediatr., 46: 40-43.

7. Marianneau, P., F. Megret, R. Olivier, D.M. Morens and V. Deubel, 1996. Dengue 1 virus binding to human hepatoma Hep G2 and simian VERO cell surfaces differs. J. Gen. Virol., 77: 2547-2554.

8. Marianneau, P., A. Cardona, L. Edelman, V. Deubel and P. Despres, 1997. Dengue virus replication in human hepatoma cells activates NF-kappaB which in turn induces apoptotic cell death. J. Virol., 71: 3244-9.

9. Lin, Y.L., C.C. Liu, J.I. Chuang, H.Y. Lei, T.M. Yeh, Y.S. Lin, Y.H. Huang and H.S. Liu, 2000. Involvement of oxidative stress, NF-IL-6 and RANTES expression in dengue-2 virus infected human liver cells. Virology 10: 114-126.
10. Schall, T. J., J.B. Jongstra, J. Dyer, J.Jorgensen, C. Clayberger, M.M. Davis and A.M. Krensky, 1988. A human T cell-specific molecule is a member of a new gene family. J. Immunol., 141: 1018-1025.

11. Cocchi, F., A.L. Devico, A. Garzino-Demo, A.K. Arya, R.C. Gallo and P. Lusso, 1995. Identification of RANTES, MIP-1 $\alpha$, and MIP-1 $\beta$ as the major HIV-suppressive factors produced by CD8 cells. Science, 270: 1811.

12. Raport, C.J., J. Gosling, V.L. Schweickart, P.W. Gray and I.F. Charo, 1996. Molecular cloning and functional characterization of a novel human CC chemokine receptor (CCR5) for RANTES, MIP-1 $\beta$, and MIP-1 $\alpha$. J. Boil. Chem., 271: 17161.

13. Kelly, M.D., H.M. Naif, S.L. Adams, A.L. Cunningham and A.R. Lloyd, 1998. Cutting edge: dichotomous effects of b-chemokines on HIV replication in monocytes and monocyte-derived macrophages. J. Immunol., 160: 3091.

14. Schall, T.J., K. Bacon, K.J. Toy and D.V. Goeddel, 1990. Selective attraction of monocytes and $\mathrm{T}$ lymphocytes of the memory phenotype by cytokine RANTES. Nature, 347: 669-671.

15. Rot, A., M. Krieger, T.S. Brunner, C. Bischoff, T. J. Schall and C. A. Dahinden, 1992. RANTES and acrophsge inflammatory protein 1 alpha induce the migration and activation of normal human eosinophil granulocytes. J. Exp. Med., 176: 1489-1495.

16. Dahinden, C.A., T. Geiser, T. Brunner, V. Von Tschamer, D. Caput, P. Ferrara, A. Minty and M. Baggiolini, 1994. Monocyte chemotactuc protein 3 is a most effective basophil- and eosinophil-activating chemokine. J. Exp. Med., 179: 751-756.

17. Taub, D.D., T.J. Sayers, C.R. Carter and J.R. Ortaldo, 1995. Alpha and beta chemokines induce $\mathrm{NK}$ cell migration and enhqance NK-mediated cytolysis. J. Immunol., 155: 3877-3888.

18. Davis, R.J., 1994. MAPKs: new JNK expand the group. Trends Biochem. Sci., 19: 470.

19. Han, J., J.D. Lee, L. Bibbs and R. J. Ulevitch, 1994. A MAP kinase targeted by endotoxin and hyperosomolarity in mammalian cells. Science, 265, 808

20. Rouse, J., S. Cohen, S. Trigon, M. Morahage, A. Alonso-Liamazares, D. Zamanillo, T. Hunt and A. R. Nebreda, 1994. A novel kinase cascade triggered by stress and heat shock that stimulates MAPKAP kinase-2 and phosphorylation of the small heat shock proteins. Cell, 78: 1027. 
21. Pandey, P., J. Raingeaud, M. Kaneki, R. Weichselbaum, R. J. Davis, D. Kufe and S. Kharband, 1996. Activation of p38 mitogen-activated protein kinase by c-abl-dependent and-independent mechanism. J. Biol Chem., 271: 23775.

22. Raingeaud, J., S. Gupta, J. S. Rogers, M. Dickens, J. Han, R. Ulevitch and R. J. Davis, 1995. Pro-inflammatory cytokines and environmental stress cause p38 mitrogen-activated protein kinase activation by dual phosphorylation on tyrosine and threonine. J. Biol. Chem., 270: 7420.

22. Hashimoto, S., K. Matsumoto, K.Y. Gon, T. Nakayama, I. Takeshita and T. Horie, 1999. Hyperosomolarity-induced IL-8 expression in human bronchial epithelial cells through p38 MAP kinase. Am. J. Respir. Crit. Care Med., 159: 634.

23. Gon, Y., S. Hashimoto, K. Matsumoto, T. Nakayama, I. Takeshita and T. Horie, 1998. Colling and rewarming-induced IL-8 expression in human bronchial epithelial cells through p38 MAP kinase-dependent pathway. Biochem. Biophys. Res. Commun., 249: 156.

24. Cowley, S., H. Paterson, P. Kemp and C.J. Marshall, 1994. Activation of MAP kinase kinase is necessary and sufficient for PC12 differentiation and for transformation of NIH 3T3 cells. Cell, 77: 841.

26. Force, T., and J.V. Bnventre, 1998. Growth factors and mitogen-activated protein kinase. Hypertension, 31: 152 .

27. Trotta, R., P. Kanakaraj and B. Perussia, 1996. Fc $\gamma$ R-dependent mitogen-activated protein kinase activation in leukocytes: a common signal transduction event necessary for expression of TNF- $\alpha$ and early activation gene. J. Exp. Med., 184: 1027.

28. Rose, D.M., B. W. Winston, E.D. Chan, D.W.H. Riches, P. Gerwins, G. L. Johnson and P. M. Henson, 1997. Fc $\gamma$ receptor cross-linking activares p42, p38, and JNK/SAPK mitogen-activated protein kinase in murine macrophages: role for p42 MAP in Fc $\gamma$ receptor-stimulated TNF- $\alpha$ synthesis. J. Immunol., 158: 3433

29. Zhang, C., R.A. Baumgartner, K. Yamada and M.A. Beaven, 1997. Mitogen-activated protein (MAP) kinase regulates production of tumor necrosis factor- $\alpha$ and release of arachidonic acid in mast cells: indication of communication between release p38 and p42 MAP kinase. J. Biol. Chem., 272:13397.
30. Bhat, N.R., P. Zhang, J.C. Lee and E.L. Hogan, 1998. Extracellular signal-regulated kinase and p38 subgroups of mitogen-activated protein kinases regulate inducible nitric oxide synthase and tumor necrosis factor- $\alpha$ gene expression in endotoxin-stimulated primary glial cultures, J. Neurosci., 18: 1633.

31. Rawadi, G., V. Ramez, B. Lemercier and S. Roman-Roman, 1998. Activation of mitogen-activated protein kinase pathways by Mycoplasma fermentans membrane lipoproteins in murine macrophages: involvement in cytokine synthesis. J. Immunol., 160: 1330.

32. Tuyt, L.M.L., W.H.A. Dokter, K. Birkenkamp, S. B. Koopmans, C. Lummen, W. Kruijer and E. Vellenga, 1999. Extracellular-regulated kinase 1/2, Jun N-terminal kinase, and c-Jun are involved in NF-кB-dependent IL-6 expression in human moncytes. J. Immunol., 162: 4893.

33. Nelson, P.J., H.T. Kim, W.C. Manning, T.J. Goralski and A. M. Krensky, 1993. Genomic organization and transcriptional regulation of the RANTES chemokine gene. J. Immunol., 151: 2601-2611.

34. Nelson, P.J., B.D. Ortiz, J.M. Pattison and A.M. Krensky, 1996. Identification of a novel regulatory region critical for expression of the RANTES chemokine in activated T lymphocytes. J. Immunol., 157: 1139-1148.

35. Moriuchi, H., M. Moriuchi and A.S. Fauci, 1997. Nuclear factor- $\pi \mathrm{B}$ potently up-regulates the promoter activity of RANTES, a chemokine that blocks HIV infection. J. Immunol., 158: 3483-3491.

36. Ortiz, B.D., P.J. Nelson and A.M. Krensky, 1997. Switching gears during T-cell maturation RANTES and late transcription. Immunol. Today, 18: 468-471.

37. Meucci, O., A. Fatatis, A.A. Simen, T.J. Bushell, P. W. Gray and R. J. Miller, 1998. Chemokines regulate hippocampal neuronal signaling and gp120 neurotoxicity. Proc. Natl. Acad. Sci. USA., 95: 14500-14505.

38. Kujime, K., S.G. Hashimoto, Y. Gon., K. Shimizu and T. Horie, 2000. p38 mitogen-activated protein kinase and c-Jun- $\mathrm{NH}_{2}$-Terminal kinase regulate RANTES production by influenza virus-infected human bronchial epithelial cells. J. Immunol., 164: 3222-3288. 
39. Matsuguchi, T., S. Okamura, C. Kawasaki and Y. Niho, 1990. Production of interleukin 6 from human liver cell lines: production of interleukin 6 is not concurrent with the production of alpha-fetoprotein. Cancer Res., 50: 7457-7459.

40. Avirutnan, P., P. Malasit, B. Seliger, S. Bhakdi and M. Husmann, 1998. Dengue virus infection of human endothelial cells leads to chemokine production, complement activation, and apoptosis. J. Immunol., 161: 6338-6346.

40. Nakajima, T., S. Kinoshita, T. Sasagawa, K. Sasaki, M. Naruto, T. Kishimoto and S. Akira, 1993. Phosphorylation at threonine-235 by a ras-dependent mitogen-activated protein kinase cascade is essential for transcription factor NF-IL-6. Proc. Natl. Acad. Sci. USA., 90:2207-2211.
41. Matsukura, S., F. Kokubu, H. Kubo, T. Tomita, H. Tokunaga, M. Kadokura, T. Yamamoto, Y. Kuroiwa, T. Ohno, H. Suzaki and M. Adachi, 1998. Expression of RANTES by normal airway epithelial cells after influenza virus A infection. Am. J. Respir. Cell Mol. Bio., 18: 255-264.

43. Thomas, L.H., J.S. Friedland, M. Sharland and S. Becker, 1998. Respiratory syncytial virus-induced RANTES production from human bronchial epithelial cells is dependent on nuclear factor- $\mathrm{kB}$ nuclear binding and is inhibited by adenovirus-mediated expression of inhibitor of $\kappa \mathrm{B} \alpha$. J. Immunol., 161: 1007-1016. 